amelioration unlikely. Secondly, the data were gathered from only a small group of patients willing to consent to two prolonged right heart catheterisation procedures 6 months apart. Nevertheless, this group was relatively homogeneous, with NYHA class III disease and high $\bar{P}_{\text {pa }}$ at presentation. Our results cannot be generalised, however, to other related $\mathrm{PAH}$ disease states, such as congenital heart disease or chronic pulmonary thromboembolism. Thirdly, our already lengthy study protocols (each lasting 2-3 h) did not allow time for cardiac output measurements at baseline and after each vasoactive drug, on each occasion of study. It is known from previous work that bosentan reduces pulmonary vascular resistance and increases cardiac output in $\mathrm{PAH}$ patients such as ours [10]; given this, the ability of ACh to produce greater vasodilatation after, compared with before, bosentan treatment clearly indicates improved endothelium-dependent dilator capacity after bosentan therapy. Finally, although IVUS measurements from control subjects were available to compare with the PAH patients, we did not perform (the relatively lengthy protocol for) pulmonary vascular reactivity studies in the healthy control subjects, to minimise risk to these volunteers.

In summary, we report, for the first time in humans, that endothelin-1 receptor antagonism improves pulmonary microvascular endothelial function in patients with severe PAH. Although further confirmatory work is required to better elucidate the mechanistic pathways involved, our study provides in vivo evidence for a mechanism by which bosentan may contribute to its therapeutic effects in PAH patients.

\section{R. Ilsar*, ${ }^{*}$, J. Levitt ${ }^{\#}$, M.K.C. $\mathrm{Ng}^{*, \#}$,L. Kritharides", M.R. Adams ${ }^{*, \#}$ and D.S. Celermajer ${ }^{*, \#}$}

*Cardiology Dept, Royal Prince Alfred Hospital, "Dept of Medicine, University of Sydney, Camperdown, and "Dept of Cardiology, Concord Repatriation General Hospital, Concord, Australia.

Correspondence: D. Celermajer, Cardiology Dept, Royal Prince Alfred Hospital, Missenden Road, Camperdown, NSW 2050, Australia. E-mail: celermajer@bigpond.com

Support Statement: This study was funded, in part, by a grant from Actelion Pharmaceuticals Australia. The authors had full control over the design, execution, interpretation and presentation of the study.

Statement of Interest: Statements of interest for R. Ilsar, L. Kritharides, D.S. Celermajer and for the study itself can be found at www.erj.ersjournals.com/site/misc/statements.xhtml

Acknowledgements: The study authors would like to thank V. Miller and D. Fowler (both Dept of Cardiology, Royal Prince Alfred Hospital, Sydney, Australia) for their assistance in patient recruitment and follow-up, and S. O'Meagher (Dept of Rheumatology, Royal Prince Alfred Hospital) for his help with data analysis.

\section{REFERENCES}

1 Farber HW, Loscalzo J. Pulmonary arterial hypertension. $N$ Engl J Med 2004; 351: 1655-1665.

2 Scott PJ, Essop AR, al-Ashab W, et al. Imaging of pulmonary vascular disease by intravascular ultrasound. Int J Card Imaging 1993; 9: 179-184.

3 Celermajer DS, Cullen S, Deanfield JE. Impairment of endothelium-dependent pulmonary artery relaxation in children with congenital heart disease and abnormal pulmonary hemodynamics. Circulation 1993; 87: 440-446.

4 Rubin LJ, Badesch DB, Barst RJ, et al. Bosentan therapy for pulmonary arterial hypertension. N Engl J Med 2002; 346: 896-903.

5 Verma S, Lovren F, Dumont AS, et al. Endothelin receptor blockade improves endothelial function in human internal mammary arteries. Cardiovasc Res 2001; 49: 146-151.

6 Pearl JM, Wellmann SA, McNamara JL, et al. Bosentan prevents hypoxia-reoxygenation-induced pulmonary hypertension and improves pulmonary function. Ann Thorac Surg 1999; 68: 1714-1721.

7 Lattimore J-DL, Wilcox I, Adams MR, et al. Treatment of obstructive sleep apnoea leads to enhanced pulmonary vascular nitric oxide release. Int J Cardiol 2008; 126: 229-233.

8 Celermajer DS, Dollery C, Burch M, et al. Role of endothelium in the maintenance of low pulmonary vascular tone in normal children. Circulation 1994; 89: 2041-2044.

9 Kielstein JT, Bode-Boger SM, Hesse G, et al. Asymmetrical dimethylarginine in idiopathic pulmonary arterial hypertension. Arterioscler Thromb Vasc Biol 2005; 25: 1414-1418.

10 Channick RN, Simonneau G, Sitbon O, et al. Effects of the dual endothelin-receptor antagonist bosentan in patients with pulmonary hypertension: a randomised placebo controlled study. Lancet 2001; 358: 1119-1123.

DOI: $10.1183 / 09031936.00083910$

\title{
Renal failure as first manifestation of familial sarcoidosis
}

\section{To the Editors:}

Sarcoidosis is a systemic illness of unknown origin characterised by the presence of epithelioid, noncaseating granulomas in multiple organs, most commonly the lungs, eyes and skin. Renal disease is uncommon; however, it is an indication to perform a diagnostic and prognostic renal biopsy, and to put the patient on treatment with high and prolonged steroid doses.
The presence of several cases in the same family is well known, as is the association with other chronic inflammatory disorders, favouring a genetic, environmental and/or immunological aetiology [1].

We report a case of a 40-yr-old male referred to the nephrologist because of high levels of creatinine. He had been a 5 pack-yr smoker until 8 yrs previous and had worked as a 
salesperson in a large store. Except for mild asthenia, polyuria and polydipsia, he remained asymptomatic. Physical examination was entirely normal. Abdominal ultrasonography showed bilateral nephrocalcinosis and spleen enlargement, and the patient was admitted for further evaluation. Red and white blood cell counts were normal. Serum chemistry showed the following (normal ranges in parentheses): creatinine $3.2 \mathrm{mg} \cdot \mathrm{dL}^{-1}$ $\left(0.7-1.3 \mathrm{mg} \cdot \mathrm{dL}^{-1}\right)$; calcium $13.5 \mathrm{mg} \cdot \mathrm{dL}^{-1}\left(8,2-10,6 \mathrm{mg} \cdot \mathrm{dL}^{-1}\right) ; 1,25-$ dihydroxyvitamin $\mathrm{D} 62,2 \mathrm{ng} \cdot \mathrm{dL}^{-1}\left(10-50 \mathrm{ng} \cdot \mathrm{dL}^{-1}\right)$; parathyroid hormone $8.7 \mathrm{pg} \cdot \mathrm{mL}^{-1}\left(11-72 \mathrm{pg} \cdot \mathrm{mL}^{-1}\right)$; angiotensine converting enzyme $178 \mathrm{IU} \cdot \mathrm{L}^{-1}\left(18-55 \mathrm{IU} \cdot \mathrm{L}^{-1}\right)$; and 24 -h urine calcium excretion $976 \mathrm{mg} \cdot \mathrm{day}^{-1}\left(10-300 \mathrm{mg} \cdot \mathrm{day}^{-1}\right)$. Thyroid hormone levels, immunoglobulin (Ig) levels and antigliadin IgA levels were normal. Chest radiography showed bilateral diffuse interstitial infiltrates and a high-resolution computed tomography scan further showed hilar, mediastinal and axilar adenopathy. There were interlobar septal thickening and peripheral and subpleural small nodules related to pulmonary vessels. A 2IU purified protein derivative RT-23 test showed no induration after $72 \mathrm{~h}$. The ECG was normal, as was the ocular exam. Pulmonary function test results were as follows: forced vital capacity (FVC) 4,670 mL (94\% predicted); forced expiratory volume in $1 \mathrm{~s}$ (FEV1) 4,110 mL (100\% pred); FEV1/FVC $88 \%$ $(110 \%)$; single-breath diffusing capacity of the lung for carbon monoxide $66 \%$; and transfer coefficient of the lung for carbon monoxide $80 \%$. Fibreoptic bronchoscopy was normal. Bronchoalveolar lavage showed 44\% lymphocytes (CD4+ $86 \%$, CD $8+12 \%$ and CD4+/CD8+ 7\%) and transbronchial biopsy revealed noncaseating granulomas with negative Lowenstein cultures. A renal biopsy was also performed, showing sclerotic changes affecting $50 \%$ of glomeruli, and interstitial fibrosis and granuloma. The patient was treated with saline solution, calcitonin, bisphosphonates and $0.5 \mathrm{mg} \cdot \mathrm{kg}^{-1}$ prednisone per day. At discharge, serum creatinine was $2.2 \mathrm{mg} \cdot \mathrm{dL}^{-1}$ and the patient was diagnosed with stage II thoracic, renal and splenic sarcoidosis with chronic renal failure due to interstitial nephritis and hypercalcaemia. A familial history was elicited (fig. 1). He had eight female siblings, one of whom had been diagnosed with sarcoidosis and coeliac disease. Two other siblings also had coeliac disease. A female cousin had been diagnosed with sarcoidosis with ocular, thoracic and central nervous system disease and Graves' disease. Pulmonary transplantation had been performed on another of his cousins due to "lung fibrosis".

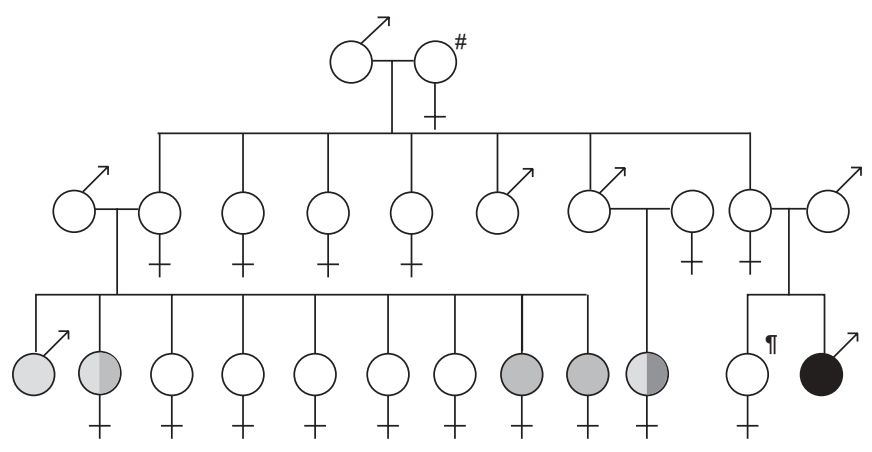

FIGURE 1. The patient's family tree showing confirmed and possible cases of sarcoidosis ( $\bullet$ ), coeliac disease $(\bullet)$, Graves' disease $(\bullet)$ and ulcerative colitis (๑). ${ }^{*}$ : died of pulmonary disease at 60 yrs of age; ": pulmonary transplantation at $45 \mathrm{yrs}$ of age because of "pulmonary fibrosis".
Ulcerative colitis was present in yet another cousin and his grandmother had died of lung disease at a young age. Prednisone was tapered and suppressed after 1 yr of treatment but had to be reinstituted because of clinical and analytical worsening. 2 yrs after diagnosis, serum creatinine levels were $2.2 \mathrm{mg} \cdot \mathrm{mL}^{-1}$, while the patient was still receiving $5 \mathrm{mg}$ of prednisone every other day.

Renal disease is uncommon in sarcoidosis and usually diagnosed after lung disease is already evident. Renal granulomas are found in 15-40\% of necropsies but renal dysfunction due solely to granulomas is considered a rare clinical finding. Three forms of renal involvement, namely, nephrocalcinosis, interstitial nephritis and glomerulonephritis, may cause renal failure. Nephrocalcinosis and nephrolithiasis, the most common causes of renal failure, are the result of an enhanced activation of $1 \alpha$-hydroxylase in sarcoid granulomas, increasing the levels of endogenous calcitriol, causing hypercalcaemia and hypercalciuria. Up to $10 \%$ of patients with sarcoidosis have hypercalcaemia. Hypercalciuria is much more common, with an estimated frequency up to $30-40 \%$. Interstitial inflammation, and occasionally fibrosis, in the absence of granuloma and nephrocalcinosis, have been noted in patients with sarcoidosis and renal failure, resulting in renal dysfunction from the activation of cellular immunity in renal tissue [2]. Glomerular disease, a less frequent cause of renal insufficiency, has been described as membranous glomerulopathy, proliferative glomerulonephritis and IgA nephropathy. Enhanced production of Igs may be involved in the pathogenesis of membranous glomerulonephritis, but neither antigen nor specific antibodies have been identified in glomerular immune deposits. Although steroid treatment is useful in any histological form of renal involvement, because renal relapse of sarcoidosis has been reported under a low-dose regime, the presence of interstitial nephritis is an indication of higher and prolonged doses of these drugs [2]. In fact, in the patient described here, prednisone had to be reinstituted after tapering because of clinical and analytical worsening. In rare cases, even treated renal disease progresses and dialysis or renal transplantation have to be performed.

The presence of several cases of sarcoidosis in the same family is a well-known occurrence. Since the first report in 1923, a total of nearly 450 cases had been presented worldwide up to 2000 [3]. By 1971, 80 cases had been reported in 35 families but only three with more than two relatives affected. The patient here described had two confirmed cases of sarcoidosis in his family, a sister and a cousin, one of them previously reported. His grandmother died at the age of $60 \mathrm{yrs}$ of an undefined pulmonary disease. A lung transplantation had been performed on another cousin because of "pulmonary fibrosis" of unknown aetiology, representing two other possible cases of sarcoidosis (fig. 1). Recently, a case-control aetiological study of sarcoidosis has shown a familial relative risk of 4.7 (95\% CI 2.3-9.7) [4]. That study showed that siblings have the highest relative risk, followed by avuncular relationships, grandparents and parents. The fact that the overall familial relative risk in siblings was larger than that in parents suggested either a recessive mode of inheritance with incomplete penetrance or a shared environmental effect [4]. Moreover, a recent study based on the ACCESS (A Case-Control Etiologic Study of Sarcoidosis) data set has suggested that sarcoidosis is due to an interaction of factors, including genetic predisposition and environmental exposure, 
in at least some cases of sarcoidosis [5]. In a review of literature, we have found that human leukocyte antigen (HLA) genotypes DR 11, 12, 13, 14, 15 and 17 have been reported to confer susceptibility to the disease, HLA DRB1 and DQ B1 have been associated with acute sarcoidosis and a good prognosis, and HLA A1, B7, B8, B13, DR3, DR5, DR14, DR15 and DR17 with familial cases $[1,6]$. A specific association between sarcoidosis and a variant of the butyrofilin-like 2 (BTNL2) gene has recently been described [7]. Clinical manifestations do not differ between sporadic and familial cases. In the same way, familial sarcoidosis does not imply a worse prognosis, with several reported cases in whom treatment is not required but spontaneous remission happens. Although the ACCESS study showed that siblings of patients with sarcoidosis are at increased risk of the disease, the phenotypic features and clinical outcomes exhibit minimal concordance, with the exception of eye and liver disease [6].

The association between immune-mediated and chronic inflammatory diseases and sarcoidosis has been described in case series. This association does not occur by chance, as a recent report establishes [8]. In that report, when all immunemediated and chronic inflammatory diseases for which associations were sought were combined, the overall rate ratio associated with sarcoidosis was 2.2 (95\% CI 1.9-2.6). Of these "overlap syndromes", Graves' disease, ulcerative colitis and coeliac disease have already been linked to sarcoidosis. Interestingly, antigliadin and antiendomysium antibodies have been found in patients with sarcoidosis, whether coexisting or not with coeliac disease [9]. A significantly higher prevalence of Graves' disease of clear autoimmune origin has been reported in female sarcoidosis patients compared with a large group of control subjects. In that report, thyroid function tests and ultrasonography were recommended as routine in female sarcoidosis patients [10]. Although it was not possible to perform either these tests or a genetic study in his siblings, we did not find a positive titre of antigliadin antibodies in this patient; nor we did find abnormal thyroid hormone levels. However, three siblings of our patient had coeliac disease, coexisting with sarcoidosis in one of them. In addition to this, one if his cousins had Graves' disease and another had ulcerative colitis, suggesting a genetic or environmental link with all these overlap syndromes.

In summary, if high levels of creatinine are found in a patient with hypercalcaemia and/or hypercalciuria, sarcoidosis must be excluded. In the same way, if high levels of creatinine are found in a patient with sarcoidosis, renal biopsy is mandatory to prove renal disease and its type. When sarcoidosis is suspected, we believe that the initial evaluation should include thyroid function tests, and assays for antigliadin and antiendomysium antibodies. Based on the familial case presented here and other previous reports, a careful familial history should be obtained, including symptoms suggesting sarcoidosis and other immunological and inflammatory diseases.

\section{E. Pastor*, J.M. Arriero", A.I. Gutiérrez*, M.E. Barroso*, R.J. Noguera", C. Muñoz ${ }^{+}$and M.L. Porriño\#}

*Respiratory Service, "Nephrology Service and ${ }^{+}$Radiology Service, Hospital de Torrevieja, and "Respiratory Service, Hospital de San Juan, Alicante, Spain.

Correspondence: E. Pastor, Respiratory Service, Hospital de Torrevieja, Ctra Torrevieja a San Miguel de Salinas, CV-95 Partida la Ceñuela, 03186 Torrevieja, Alicante, Spain. E-mail: pastor_estesp@gva.es

Statement of Interest: None declared.

\section{REFERENCES}

1 Statement on sarcoidosis. Joint Statement of the American Thoracic Society (ATS), the European Respiratory Society (ERS) and the World Association of Sarcoidosis and Other Granulomatous disorders (WASOG) adopted by the ATS Board of Directors and by the ERS Executive Committee, February 1999. Am J Respir Crit Care Med 1999; 160: 736-755.

2 Muther RS, McCarron DA, Bennett WM. Renal manifestations of sarcoidosis. Arch Intern Med 1981; 141: 643-645.

3 Elford J, Fitch P, Kaminski E, et al. Five cases of sarcoidosis in one family: a new immunological link? Thorax 2000; 55: 343-344.

4 Rybicki BA, Iannuzzi MC, Frederick MM, et al. Familial aggregation of sarcoidosis. A case-control etiologic study of sarcoidosis (ACCESS). Am J Respir Crit Care Med 2001; 164: 2085-2091.

5 Rossman MD, Thompson B, Frederick M, et al. HLA and environmental interactions in sarcoidosis. Sarcoidosis Vasc Diffuse Lung Dis 2008; 25: 125-132.

6 Iannuzzi MC, Rybicki BA, Teirstein AS. Sarcoidosis. N Engl J Med 2007; 357: 2153-2165.

7 Valentonyte R, Hampe J, Huse K, et al. Sarcoidosis is associated with a truncating splice site mutation in BTNL2. Nat Genet 2005; 37: 357-364.

8 Rajoriya N, Wotton CJ, Yeates DG, et al. Immune-mediated and chronic inflammatory disease in people with sarcoidosis: disease associations in a large UK database. Postgrad Med J 2009; 85: 233-237.

9 Papadopoulos KI, Sjöberg K, Lindgren S, et al. Evidence of gastrointestinal immune reactivity in patients with sarcoidosis. J Intern Med 1999; 245: 525-531.

10 Antonelli A, Fazzi P, Fallahi P, et al. Prevalence of hypothyroidism and Graves disease in sarcoidosis. Chest 2006; 130: 526-532.

DOI: $10.1183 / 09031936.00077710$ 